\title{
A $(P, V)$-EXTENSION OF HURWITZ-LERCH ZETA FUNCTION AND ITS PROPERTIES
}

\author{
GAUHAR RAHMAN ${ }^{1}$, KOTTAKKARAN SOOPPY NISAR $^{2, \star}$ AND SHAHID MUBEEN $^{3}$
}

${ }^{1}$ G. Rahman: Department of Mathematics, International Islamic University, Islamabad, Pakistan, email: gauhar55uom@gmail.com

${ }^{2}$ Department of Mathematics, College of Arts \& Science-Wadi Addawaser, Prince Sattam bin Abdulaziz University, Saudi Arabia, email: n.sooppy@psau.edu.sa

${ }^{3}$ Shahid Mubeen: Department of Mathematics, University of Sargodha, Sargodha, Pakistan, email: smjhanda@gmail.com

* Corresponding Author.

AbStract. In this paper, we define a $(p, v)$-extension of Hurwitz-Lerch Zeta function by considering an extension of beta function defined by Parmar et al. [J. Classical Anal. 11 (2017) 81106]. We obtain its basic properties which include integral representations, Mellin transformation, derivative formulas and certain generating relations. Also, we establish the special cases of the main results.

keywords: Extended beta function, hypergeometric functions, Hurwitz-Lerch Zeta functions, Mellin transform, transformation formula and summation formula.

MSC[2010]: 11B68, 33C20, 33C45, 33C65, 33C05

\section{INTRODUCTION}

The Hurwitz-Lerch Zeta function and its properties are appear in various recent research papers (see e.g., [10] pp. 27, [24], pp. 121 and [25], pp. 194). The definition and integral representation of Hurwitz-Lerch Zeta function respectively given by

$$
\begin{gathered}
\Phi(z, \sigma, a)=\sum_{n=0}^{\infty} \frac{z^{n}}{(n+a)^{\sigma}}, \\
\left(a \in \mathbb{C} \backslash \mathbb{Z}_{0}^{-}, s \in \mathbb{C} \text {, when }|z|<1 ; \Re(\sigma)>1 \text { when }|z|=1\right) . \\
\Phi(z, \sigma, a)=\frac{1}{\Gamma(\sigma)} \int_{0}^{\infty} \frac{t^{\sigma-1} e^{-a t}}{1-z e^{-t}} d t=\frac{1}{\Gamma(\sigma)} \int_{0}^{\infty} \frac{t^{\sigma-1} e^{-(a-1) t}}{e^{t}-z} d t \\
(\Re(\sigma)>0, \Re(a)>0, \text { when }|z| \leq 1(z \neq 1) ; \Re(\sigma)>1 \text { when } z=1) .
\end{gathered}
$$

Many extension and generalizations of (1.1) found in the literature (see .g., [3, 7, 10, 11]). In [13], authors gave the following generalization and integral representation:

$$
\begin{gathered}
\Phi_{\zeta}^{*}(z, \sigma, a)=\sum_{n=0}^{\infty} \frac{(\zeta)_{n}}{n !} \frac{z^{n}}{(n+a)^{\sigma}}, \\
\left(\zeta \in \mathbb{C}, a \in \mathbb{C} \backslash \mathbb{Z}_{0}^{-}, s \in \mathbb{C}, \text { when }|z|<1, \Re(\sigma-\zeta)>1 \text { when }|z|=1\right) . \\
\Phi_{\zeta}^{*}(z, \sigma, a)=\frac{1}{\Gamma(\sigma)} \int_{0}^{\infty} \frac{t^{\sigma-1} e^{-a t}}{\left(1-z e^{-t}\right)^{\zeta}} d t=\frac{1}{\Gamma(\sigma)} \int_{0}^{\infty} \frac{t^{\sigma-1} e^{-(a-\zeta) t}}{\left(e^{t}-z\right)^{\zeta}} d t \\
(\Re(\sigma)>0, \Re(a)>0, \text { when }|z| \leq 1(z \neq 1) ; \Re(\sigma)>1 \text { when } z=1) .
\end{gathered}
$$

The extension of (1.3) and its integral representation are given in [12]:

$$
\Phi_{\varrho, \varsigma ; \zeta}(z, \sigma, a)=\sum_{n=0}^{\infty} \frac{(\varrho)_{n}(\varsigma)_{n}}{(\zeta)_{n} n !} \frac{z^{n}}{(n+a)^{\sigma}},
$$


$\left(\varrho, \varsigma, \zeta \in \mathbb{C}, a \in \mathbb{C} \backslash \mathbb{Z}_{0}^{-}, s \in \mathbb{C}\right.$, when $|z|<1, \Re(\sigma+\zeta-\varrho-\varsigma)>1$ when $\left.|z|=1\right)$.

$$
\begin{gathered}
\Phi_{\varrho, \varsigma ; \zeta}(z, \sigma, a)=\frac{1}{\Gamma(\sigma)} \int_{0}^{\infty} t^{\sigma-1} e^{-a t}{ }_{2} F_{1}\left(\varrho, \varsigma ; \zeta ; z e^{-t}\right) d t \\
(\Re(\sigma)>0, \Re(a)>0 \text {, when }|z| \leq 1(z \neq 1) ; \Re(\sigma)>1 \text { when } z=1) .
\end{gathered}
$$

In [21], more generalization and integral representation are given in the following form,

$$
\Phi_{\varrho, \varsigma ; \zeta}(z, \sigma, a ; p)=\sum_{n=0}^{\infty} \frac{(\varrho)_{n} B(\varsigma+n, \zeta-\varsigma ; p)}{B(\varsigma, \zeta-\varsigma) n !} \frac{z^{n}}{(n+a)^{\sigma}},
$$

where $\left(p \geq 0, \varrho, \varsigma, \zeta \in \mathbb{C}, a \in \mathbb{C} \backslash \mathbb{Z}_{0}^{-}, s \in \mathbb{C}\right.$ when $|z|<1, \Re(\sigma+\zeta-\varrho-\varsigma)>1$, when $\left.|z|=1\right)$ and

$$
\Phi_{\varrho, \varsigma ; \zeta}(z, \sigma, a)=\frac{1}{\Gamma(\sigma)} \int_{0}^{\infty} t^{\sigma-1} e^{-a t} F_{p}\left(\varrho, \varsigma ; \zeta ; z e^{-t}\right) d t
$$

where $p \geq 0, \Re(\sigma)>0 \Re(a)>0$, when $|z| \leq 1(z \neq 1) ; \Re(\sigma)>1$ when $z=1$, and $F_{p}$ is the extended hypergeometric function (see [5]).

Now, we recall the following:

The extended beta function $B\left(\delta_{1}, \delta_{2} ; p\right)$ due to [4] is

$$
B\left(\delta_{1}, \delta_{2} ; p\right)=B_{p}\left(\delta_{1}, \delta_{2}\right)=\int_{0}^{1} t^{\delta_{1}-1}(1-t)^{\delta_{2}-1} e^{-\frac{p}{t(1-t)}} d t
$$

where $\Re(p)>0, \Re(x)>0, \Re(y)>0$ respectively. When $p=0$, then $B\left(\delta_{1}, \delta_{2} ; 0\right)=B\left(\delta_{1}, \delta_{2}\right)$.

In [23] Parmar et al. defined extended beta function as:

$$
B_{v}\left(\delta_{1}, \delta_{2} ; p\right)=B\left(\delta_{1}, \delta_{2} ; p, v\right)=\sqrt{\frac{2 p}{\pi}} \int_{0}^{1} t^{\delta_{1}-\frac{3}{2}}(1-t)^{\delta_{2}-\frac{3}{2}} K_{v+\frac{1}{2}}\left(\frac{p}{t(1-t)}\right) d t
$$

where $\Re(x)>0, \Re(y)>0$ and $K_{v+\frac{1}{2}}($.$) is modified Bessel function of order v$. Obviously, when $v=0$ then $B_{0}\left(\delta_{1}, \delta_{2} ; p\right)=B\left(\delta_{1}, \delta_{2} ; p\right)$ is the extended beta function (see[4]). They also defined the extended hypergeometric function and its integral representation as

$$
\begin{aligned}
F_{v}\left(\sigma_{1}, \sigma_{2} ; \sigma_{3} ; z ; p\right)={ }_{2} F_{1}\left(\sigma_{1}, \sigma_{2} ; \sigma_{3} ; z ; p, v\right) & =\sum_{n=0}^{\infty}\left(\sigma_{1}\right)_{n} \frac{B_{v}\left(\sigma_{2}+n, \sigma_{3}-\sigma_{2} ; p\right)}{B\left(\sigma_{2}, \sigma_{3}-\sigma_{2}\right)} \frac{z^{n}}{n !} \\
& =\sum_{n=0}^{\infty}\left(\sigma_{1}\right)_{n} \frac{B\left(\sigma_{2}+n, \sigma_{3}-\sigma_{2} ; p, v\right)}{B\left(\sigma_{2}, \sigma_{3}-\sigma_{2}\right)} \frac{z^{n}}{n !}
\end{aligned}
$$

where $p, v \geq 0, \sigma_{1}, \sigma_{2}, \sigma_{3} \in \mathbb{C}$ and $|z|<1$.

The use of (1.10) found in [8, 9, 16]. Very recently, Gauhar et al. [15], introduced a new extension of Hurwitz-Lerch Zeta function which is defined by

$$
\begin{aligned}
\Phi_{\varrho, \varsigma ; \zeta}(z, \sigma, a ; p, \lambda) & =\Phi_{\varrho, \varsigma ; \zeta}^{\lambda}(z, \sigma, a ; p) \\
= & \sum_{n=0}^{\infty} \frac{(\varrho)_{n} B(\varsigma+n, \zeta-\varsigma ; p, \lambda)}{B(\varsigma, \zeta-\varsigma) n !} \frac{z^{n}}{(n+a)^{\sigma}} \\
& =\sum_{n=0}^{\infty} \frac{(\varrho)_{n} B_{p}^{\lambda}(\varsigma+n, \zeta-\varsigma)}{B(\varsigma, \zeta-\varsigma) n !} \frac{z^{n}}{(n+a)^{\sigma}}
\end{aligned}
$$

$p \geq 0, \lambda>0, \varrho, \varsigma, \zeta \in \mathbb{C}, a \in \mathbb{C} \backslash \mathbb{Z}_{0}^{-}, \sigma \in \mathbb{C}$ when $|z|<1, \Re(\sigma+\zeta-\varrho-\varsigma)>1$ when $|z|=1$ where $B_{p}^{\lambda}\left(\delta_{1}, \delta_{2}\right)$ is an extended beta function defined in [26] by

$$
B_{p}^{\lambda}\left(\delta_{1}, \delta_{2}\right)=B\left(\delta_{1}, \delta_{2} ; p, \lambda\right)=\int_{0}^{1} t^{\delta_{1}-1}(1-t)^{\delta_{2}-1} E_{\lambda}\left(-\frac{p}{t(1-t)}\right) d t
$$


A $(P, V)$-EXTENSION OF HURWITZ-LERCH ZETA FUNCTION AND ITS PROPERTIES

where $\Re(x)>0, \Re(y)>0$ and $E_{\lambda}($.$) is Mittag-Leffler function defined by$

$$
E_{\lambda}(z)=\sum_{n=0}^{\infty} \frac{z^{n}}{\Gamma(\lambda n+1)}
$$

For various extensions and generalizations of special functions the readers are refer to the recent work (see e.g., $[1,2,6,14,17,19,20,22]$ ).

\section{2. $(p, v)$-Extension of Hurwitz-Lerch Zeta Function AND its Properties}

This section introduce a new extension in terms of extended beta function (1.10) called the $(p, v)$ Extension of Hurwitz-Lerch Zeta function and is defined by

$$
\begin{aligned}
\Phi_{v, \varrho, \varsigma ; \zeta}(z, \sigma, a ; p) & =\Phi_{\varrho, \varsigma ; \zeta}(z, \sigma, a ; p, v) \\
= & \sum_{n=0}^{\infty} \frac{(\varrho)_{n} B(\varsigma+n, \zeta-\varsigma ; p, v)}{B(\varsigma, \zeta-\varsigma) n !} \frac{z^{n}}{(n+a)^{\sigma}} \\
= & \sum_{n=0}^{\infty} \frac{(\varrho)_{n} B_{v}(\varsigma+n, \zeta-\varsigma ; p)}{B(\varsigma, \zeta-\varsigma) n !} \frac{z^{n}}{(n+a)^{\sigma}}
\end{aligned}
$$

$p, v \geq 0, \varrho, \varsigma, \zeta \in \mathbb{C}, a \in \mathbb{C} \backslash \mathbb{Z}_{0}^{-}, s \in \mathbb{C}$ when $|z|<1, \Re(\sigma+\zeta-\varrho-\varsigma)>1$ when $|z|=1$.

Remark 2.1. The following special cases can be derived from (2.1).

Case 1. If we consider $\varrho=1$, then (2.1) will lead to another extension of generalized Hurwitz-Lerch Zeta function $\Phi_{v, \varsigma ; \zeta}^{1,1}(z, \sigma, a ; p)$ introduced earlier in [18] with $\zeta=\sigma=1$.

$$
\begin{aligned}
\Phi_{v, \varsigma ; \zeta}^{1,1}(z, \sigma, a ; p) & =\Phi_{v, 1, \varsigma ; \zeta}(z, \sigma, a ; p) \\
= & \sum_{n=0}^{\infty} \frac{B_{v}(\varsigma+n, \zeta-\varsigma ; p)}{B(\varsigma, \zeta-\varsigma) n !} \frac{z^{n}}{(n+a)^{\sigma}}
\end{aligned}
$$

$p, v \geq 0, \varsigma, \zeta \in \mathbb{C}, a \in \mathbb{C} \backslash \mathbb{Z}_{0}^{-}, s \in \mathbb{C}$ when $|z|<1, \Re(\sigma+\zeta-\varrho-\varsigma)>1$ when $|z|=1$.

Case 2. If we set $\varrho=\zeta=1$ in (2.1), then we get a new extension of Hurwitz-Lerch Zeta function $\Phi_{v, \varsigma}^{*}(z, \sigma, a ; p)$ which is the extension defined in [13] as:

$$
\begin{aligned}
\Phi_{v, \varsigma}^{*}(z, \sigma, a ; p) & =\Phi_{v, 1, \varsigma ; 1}(z, \sigma, a ; p) \\
= & \sum_{n=0}^{\infty} \frac{B_{v}(\varsigma+n, 1-\varsigma ; p)}{B(\varsigma, 1-\varsigma) n !} \frac{z^{n}}{(n+a)^{\sigma}}
\end{aligned}
$$

$p, v \geq 0, \varsigma \in \mathbb{C}, a \in \mathbb{C} \backslash \mathbb{Z}_{0}^{-}, s \in \mathbb{C}$ when $|z|<1, \Re(\sigma+\zeta-\varrho-\varsigma)>1$ when $|z|=1$.

Case 3. A limit case of extended Hurwitz-Lerch Zeta function $\Phi_{v, \varrho, \varsigma ; \zeta}(z, \sigma, a ; p)$ defined by $(2.1)$ is given by

$$
\begin{aligned}
\Phi_{v, \varsigma ; \zeta}^{*}(z, \sigma, a ; p) & =\lim _{|\varrho| \rightarrow \infty}\left\{\Phi_{v, \varrho, \varsigma ; \zeta}\left(\frac{z}{\varrho}, \sigma, a ; p\right)\right\} \\
= & \sum_{n=0}^{\infty} \frac{B_{v}(\varsigma+n, \zeta-\varsigma ; p)}{B(\varsigma, \zeta-\varsigma) n !} \frac{z^{n}}{(n+a)^{\sigma}}
\end{aligned}
$$

$p, v \geq 0, \varsigma \in \mathbb{C}, a \in \mathbb{C} \backslash \mathbb{Z}_{0}^{-}, s \in \mathbb{C}$ when $|z|<1, \Re(\sigma+\zeta-\varrho-\varsigma)>1$ when $|z|=1$.

Remark 2.2. It is clear that, if $v=0$ then (2.1) will reduces (1.7)

$$
\begin{aligned}
\Phi_{0, \varrho, \varsigma ; \zeta}(z, \sigma, a ; p) & =\Phi_{\varrho, \varsigma ; \zeta}(z, \sigma, a ; p) \\
= & \sum_{n=0}^{\infty} \frac{(\varrho)_{n} B(\varsigma+n, \zeta-\varsigma ; p)}{B(\varsigma, \zeta-\varsigma) n !} \frac{z^{n}}{(n+a)^{\sigma}}
\end{aligned}
$$


$p, v \geq 0, \varrho, \varsigma, \zeta \in \mathbb{C}, a \in \mathbb{C} \backslash \mathbb{Z}_{0}^{-}, s \in \mathbb{C}$ when $|z|<1, \Re(\sigma+\zeta-\varrho-\varsigma)>1$ when $|z|=1$. Similarly for $p=0,(2.5)$ converts to (1.5) and for $\varrho=\zeta=1$ and $p=0$, (2.5) turns to (1.3). Taking $\varrho=\varsigma=\zeta=1$ and $p=0$ in $(2.5)$ results $(1.1)$.

\section{INTEGRAL REPRESENTATIONS AND DERIVATIVE FORMULAS}

In this section, we define various integral representations and a derivative formula for (2.1).

Theorem 3.1. The following integral representation holds true:

$$
\begin{gathered}
\Phi_{v, \varrho, \varsigma ; \zeta}(z, \sigma, a ; p)=\frac{1}{\Gamma(\sigma)} \int_{0}^{\infty} t^{\sigma-1} e^{-a t} F_{v}\left(\varrho, \varsigma ; \zeta ; z e^{-t} ; p\right) d t \\
\left(p \geq 0, \lambda>0, \varsigma \in \mathbb{C}, a \in \mathbb{C} \backslash \mathbb{Z}_{0}^{-}, s \in \mathbb{C} \text { when }|z|<1, \Re(\sigma+\zeta-\varrho-\varsigma)>1 \text { when }|z|=1\right) .
\end{gathered}
$$

Proof. Consider the Eulerian integral

$$
\frac{1}{(n+a)^{\sigma}}=\frac{1}{\Gamma(\sigma)} \int_{0}^{\infty} t^{\sigma-1} e^{-(n+a) t} d t
$$

where $\min \{\Re(\sigma), \Re(a)\}>0 ; n \in \mathbb{N}_{0}$ in $(2.1)$, we have

$$
\Phi_{v, \varrho, \varsigma ; \zeta}(z, \sigma, a ; p)=\sum_{n=0}^{\infty} \frac{(\varrho)_{n} B_{v}(\varsigma+n, \zeta-\varsigma ; p)}{B(\varsigma, \zeta-\varsigma) n !}\left(\frac{1}{\Gamma(\sigma)} \int_{0}^{\infty} t^{\sigma-1} e^{-(n+a) t} d t\right) .
$$

Interchanging the order of summation and integration under the suitable conditions given in 3.1, we have

$$
\Phi_{v, \varrho, \varsigma ; \zeta}(z, \sigma, a ; p, \lambda)=\frac{1}{\Gamma(\sigma)} \int_{0}^{\infty} t^{\sigma-1} e^{-(n+a) t}\left(\sum_{n=0}^{\infty} \frac{(\varrho)_{n} B_{v}(\varsigma+n, \zeta-\varsigma ; p)}{B(\varsigma, \zeta-\varsigma) n !}\right) d t
$$

by using (1.11) we get the desired result.

Theorem 3.2. The following formula holds true:

$$
\begin{gathered}
\Phi_{v, \varrho, \varsigma ; \zeta}(z, \sigma, a ; p)=\frac{1}{B(\varsigma, \zeta-\varsigma)} \int_{0}^{\infty} \frac{x^{\varsigma-1}}{(1+x)^{\zeta}} K_{v+\frac{1}{2}}\left(p\left(2+x+\frac{1}{x}\right)\right) \Phi_{\varrho}^{*}\left(\frac{z x}{1+x}, \sigma, a\right) d x \\
(p \geq 0, \lambda>0, \Re(\zeta)>\Re(\varsigma)>0)
\end{gathered}
$$

and

$$
\begin{aligned}
\Phi_{v, \varrho, \varsigma ; \zeta}(z, \sigma, a ; p)= & \frac{1}{B(\varsigma, \zeta-\varsigma)} \int_{0}^{\infty} \frac{t^{\sigma-1} e^{-a t} x^{\varsigma-1}}{(1+x)^{\zeta}} K_{v+\frac{1}{2}}\left(p\left(2+x+\frac{1}{x}\right)\right)\left(1-\frac{z x e^{-t}}{1+x}\right)^{-\varrho} d x \\
& (p \geq 0, \lambda>0, \Re(\zeta)>\Re(\varsigma)>0, \min \{\Re(\sigma), \Re(a)\}>0),
\end{aligned}
$$

provided that both the integrals converges.

Proof. Consider the following (see [21])

$$
B_{v}\left(\sigma_{1}, \sigma_{2} ; p\right)=\int_{0}^{\infty} \frac{x^{\sigma_{1}-1}}{(1+x)^{\sigma_{1}+\sigma_{2}}} K_{v+\frac{1}{2}}\left(p\left(2+x+\frac{1}{x}\right)\right) d x, \Re(p)>0 .
$$

Substituting $\sigma_{1}=\varsigma+n$ and $\sigma_{2}=\zeta-\varsigma$ in (3.6), we have

$$
B_{v}(\varsigma+n, \zeta-\varsigma ; p)=\int_{0}^{\infty} \frac{x^{\varsigma+n-1}}{(1+x)^{\zeta+n}} K_{v+\frac{1}{2}}\left(p\left(2+x+\frac{1}{x}\right)\right) d x, \Re(p)>0 .
$$

Using (3.7) in (2.1), we have

$$
\Phi_{v, \varrho, \varsigma ; \zeta}(z, \sigma, a ; p)=\frac{1}{B(\varsigma, \zeta-\varsigma)} \int_{0}^{\infty} \frac{x^{\varsigma-1}}{(1+x)^{\zeta}} K_{v+\frac{1}{2}}\left(p\left(2+x+\frac{1}{x}\right)\right)\left(\sum_{n=0}^{\infty} \frac{(\varrho)_{n}}{n !} \frac{(x z)^{n}}{(1+x)^{n}(n+a)^{\sigma}}\right) d x
$$

By using (1.3), we get the required result (3.4).

Now from (3.1) and (3.8), we have

$$
\Phi_{v, \varrho, \varsigma ; \zeta}(z, \sigma, a ; p)
$$


A $(P, V)$-EXTENSION OF HURWITZ-LERCH ZETA FUNCTION AND ITS PROPERTIES

$$
\begin{aligned}
& =\frac{1}{\Gamma(\sigma)} \int_{0}^{\infty} t^{\sigma-1} e^{-(n+a) t}\left(\sum_{n=0}^{\infty} \frac{(\varrho)_{n} B_{v}(\varsigma+n, \zeta-\varsigma ; p)}{B(\varsigma, \zeta-\varsigma) n !}\right) d t \\
& =\frac{1}{\Gamma(\sigma) B(\varsigma, \zeta-\varsigma)} \int_{0}^{\infty} \int_{0}^{\infty} \frac{x^{\varsigma-1} t^{\sigma-1} e^{-a t}}{(1+x)^{\zeta}} K_{v+\frac{1}{2}}\left(p\left(2+x+\frac{1}{x}\right)\right)\left(\sum_{n=0}^{\infty} \frac{(\varrho)_{n}}{n !} \frac{\left(x z e^{-t}\right)^{n}}{(1+x)^{n}(n+a)^{\sigma}}\right) d t d x .
\end{aligned}
$$

By using the binomial expansion

$$
(1-z t)^{-\alpha}=\sum_{n=0}^{\infty} \frac{(\alpha)_{n}(z t)^{n}}{n !}
$$

we get the desired result.

Theorem 3.3. The following formula holds true:

$$
\Phi_{v, \varrho, \varsigma ; \zeta}(z, \sigma, a ; p)=\frac{1}{\Gamma(\varrho)} \int_{0}^{\infty} t^{\varrho-1} e^{-t} \Phi_{\varsigma ; \zeta}^{*}(z t, \sigma, a ; p) d t
$$

$(p \geq 0, \lambda>0, \Re(\varrho)>0, \Re(a)>0$, when $|z| \leq 1(z \neq 1) ; \Re(\sigma)>1$, when $z=1)$, where $\Phi_{v, \varsigma ; \zeta}^{*}(z t, \sigma, a ; p)$ is the limiting case defined by (2.4).

Proof. Using the integral representation of Pochhammer symbol $(\varrho)_{n}$ given in [25]:

$$
(\varrho)_{n}=\frac{1}{\Gamma(\varrho)} \int_{0}^{\infty} t^{\varrho+n-1} e^{-t} d t
$$

in (2.1) and interchanging the order of summation and integration under the suitable convergence conditions, we get

$$
\Phi_{v, \varrho, \varsigma ; \zeta}(z, \sigma, a ; p, \lambda)=\frac{1}{\Gamma(\varrho)} \int_{0}^{\infty} t^{\varrho-1} e^{-t} \sum_{n=0}^{\infty} \frac{B_{v}(\varsigma+n, \zeta-\varsigma ; p)}{B(\varsigma, \zeta-\varsigma)} \frac{(z t)^{n}}{n !(n+a)^{\sigma}} d t
$$

Now, by using (2.4), we get the desired proof.

Next, we derive the derivative formula of $(2.1)$.

Theorem 3.4. The following formula holds true

$$
\frac{d^{n}}{d z^{n}}\left\{\Phi_{v, \varrho, \varsigma ; \zeta}(z, \sigma, a ; p)\right\}=\frac{(\varrho)_{n}(\varsigma)_{n}}{(\zeta)_{n}} \Phi_{v, \varrho+n, \varsigma+n ; \zeta+n}(z, \sigma, a+n ; p),\left(n \in \mathbb{N}_{0}\right)
$$

Proof. Differentiation of (2.1) with respect to $z$ yields

$$
\frac{d}{d z}\left\{\Phi_{v, \varrho, \varsigma ; \zeta}(z, \sigma, a ; p)\right\}=\sum_{n=1}^{\infty} \frac{(\varrho)_{n}}{(n-1) !} \frac{B_{v}(\varsigma+n, \zeta-\varsigma ; p)}{B(\varsigma, \zeta-\varsigma)} \frac{z^{n-1}}{(n+a)^{\sigma}} .
$$

Replacing $n$ by $n+1$ and applying the identities

$$
B(b, c-b)=\frac{c}{b} B(b+1, c-b) \text { and }(a)_{n+1}=a(a+1)_{n}
$$

in the r.h.s of (3.15), we get

$$
\frac{d}{d z}\left\{\Phi_{v, \varrho, \varsigma ; \zeta}(z, \sigma, a ; p)\right\}=\frac{\varrho \varsigma}{\zeta} \Phi_{v, \varrho+1, \varsigma+1 ; \zeta+1}(z, \sigma, a+1 ; p) .
$$

Again, differentiating (3.16) with respect to $z$, we obtain

$$
\frac{d^{2}}{d z^{2}}\left\{\Phi_{v, \varrho, \varsigma ; \zeta}(z, \sigma, a ; p)\right\}=\frac{\varrho(\varrho+1) \varsigma(\varsigma+1)}{\zeta(\zeta+1)} \Phi_{v, \varrho+2, \varsigma+2 ; \zeta+2}(z, \sigma, a+2 ; p) .
$$

Continuing up to $n$-times gives the required proof. 


\section{Mellin Transformation and generating Relations}

In this section, we define Mellin transformation and some generating relations for (2.1). Here, we recall the following:

$$
\mathfrak{M}\{f(t) ; t \rightarrow r\}=\int_{0}^{\infty} t^{r-1} f(t) d t .
$$

Theorem 4.1. The Mellin transform of (2.1) is given by

$$
\begin{gathered}
\mathfrak{M}\left\{\Phi_{v, \varrho, \varsigma ;}(z, \sigma, a ; p) ; p \rightarrow r\right\}=\frac{2^{r-1}}{\sqrt{\pi}} \Gamma\left(\frac{r-v}{2}\right) \Gamma\left(\frac{r+v+1}{2}\right) \frac{B(\varsigma+r, \zeta-\varsigma+r)}{B(\varsigma, \zeta-\varsigma)} \Phi_{\varrho, \varsigma+r ; \zeta+2 r}(z, \sigma, a) \\
(\Re(r)>0 \text { and } \Re(\varsigma+r)>0) .
\end{gathered}
$$

Proof. The Mellin Transformation of (2.1) is given by:

$$
\begin{aligned}
\mathfrak{M}\left\{\Phi_{v, \varrho, \varsigma ; \zeta}(z, \sigma, a ; p) ; p \rightarrow r\right\} & =\int_{0}^{\infty} p^{r-1} \Phi_{v, \varrho, \varsigma ; \zeta}(z, \sigma, a ; p) d p \\
& =\int_{0}^{\infty} p^{r-1}\left(\sum_{n=0}^{\infty} \frac{(\varrho)_{n}}{n !} \frac{B_{v}(\varsigma+n, \zeta-\varsigma ; p)}{B(\varsigma, \zeta-\varsigma)} \frac{z^{n}}{(n+a)^{\sigma}}\right) d p \\
& =\frac{1}{B(\varsigma, \zeta-\varsigma)} \sum_{n=0}^{\infty} \frac{(\varrho)_{n}}{n !} \frac{z^{n}}{(n+a)^{\sigma}} \\
& \times \int_{0}^{\infty} p^{r-1} B_{v}(\varsigma+n, \zeta-\varsigma ; p) d p
\end{aligned}
$$

Applying the result (see [23]),

$$
\int_{0}^{\infty} p^{r-1} B_{v}\left(\delta_{1}, \delta_{2} ; p\right) d p=\frac{2^{r-1}}{\sqrt{\pi}} \Gamma\left(\frac{r-v}{2}\right) \Gamma\left(\frac{r+v+1}{2}\right) B\left(\delta_{1}+r, \delta_{2}+r\right),
$$

we get

$$
\begin{aligned}
& \mathfrak{M}\left\{\Phi_{v, \varrho, \varsigma ; \zeta}(z, \sigma, a ; p) ; p \rightarrow r\right\} \\
= & \frac{2^{r-1}}{\sqrt{\pi}} \Gamma\left(\frac{r-v}{2}\right) \Gamma\left(\frac{r+v+1}{2}\right) \sum_{n=0}^{\infty} \frac{(\varrho)_{n}}{n !} \frac{z^{n}}{(n+a)^{\sigma}} \frac{B(\varsigma+n+r, \zeta+r-\varsigma)}{B(\varsigma, \zeta-\varsigma)} \\
= & \frac{2^{r-1}}{\sqrt{\pi}} \Gamma\left(\frac{r-v}{2}\right) \Gamma\left(\frac{r+v+1}{2}\right) \frac{B(\varsigma+r, \zeta-\varsigma+r)}{B(\varsigma, \zeta-\varsigma)} \sum_{n=0}^{\infty} \frac{(\varrho)_{n}(\varsigma+r)_{n}}{(\zeta+2 r)_{n}} \frac{z^{n}}{n !(n+a)^{\sigma}}
\end{aligned}
$$

which by using (1.5) yields the required proof.

Remark 4.1. The special case of (2.1) for $r=1$ gives the following integral representation

$$
\int_{0}^{\infty} \Phi_{\varrho, \varsigma ; \zeta}(z, \sigma, a ; p, \lambda) d p=\frac{1}{\sqrt{\pi}} \Gamma\left(\frac{1-v}{2}\right) \Gamma\left(\frac{v+2}{2}\right) \frac{B(\varsigma+1, \zeta-\varsigma+1)}{B(\varsigma, \zeta-\varsigma)} \Phi_{\varrho, \varsigma+1 ; \zeta+2}(z, \sigma, a)
$$

Corollary 4.1. The following Mellin transform of the extended Hurwitz-Lerch Zeta function is given in ( see e.g., [21]) by

$$
\begin{gathered}
\mathfrak{M}\left\{\Phi_{\varrho, \varsigma ; \zeta}(z, \sigma, a ; p) ; p \rightarrow r\right\}=\frac{\Gamma(r) B(\varsigma+r, \zeta-\varsigma+r)}{B(\varsigma, \zeta-\varsigma)} \Phi_{\varrho, \varsigma+r ; \zeta+2 r}(z, \sigma, a) \\
(\Re(r)>0 \text { and } \Re(\varsigma+r)>0) .
\end{gathered}
$$

Theorem 4.2. The following generating function for (2.1) holds true:

$$
\begin{gathered}
\sum_{n=0}^{\infty}(\varrho)_{n} \Phi_{v, \varrho+n, \varsigma ; \zeta}(z, \sigma, a ; p) \frac{t^{n}}{n !}=(1-t)^{-\varrho} \Phi_{v, \varrho+n, \varsigma ; \zeta}\left(\frac{z}{1-t}, \sigma, a ; p\right) \\
(p, v \geq 0 \varrho, \varsigma, \zeta \in \mathbb{C} \text { and }|t|<1) .
\end{gathered}
$$


Proof. Let the left hand side of (4.8) be denoted by $S_{1}$, the from (2.1), we have

$$
S_{1}=\sum_{n=0}^{\infty}(\varrho)_{n}\left\{\sum_{k=0}^{\infty}(\varrho+n)_{k} \frac{B_{v}(\varsigma+k, \zeta-\varsigma ; p)}{B(\varsigma, \zeta-\varsigma)} \frac{z^{k}}{k !(k+a)^{\sigma}}\right\} \frac{t^{n}}{n !}
$$

Interchanging the order of summations and using the identity $(\varrho)_{n}(\varrho+n)_{k}=(\varrho)_{k}(\varrho+k)_{n}$ in $(4.9)$, we have

$$
S_{1}=\sum_{k=0}^{\infty}(\varrho)_{k} \frac{B_{v}(\varsigma+k, \zeta-\varsigma ; p)}{B(\varsigma, \zeta-\varsigma)}\left\{\sum_{n=0}^{\infty}(\varrho+k)_{n} \frac{t^{n}}{n !}\right\} \frac{z^{k}}{k !(k+a)^{\sigma}}
$$

Now, using the following binomial expansion

$$
(1-t)^{-\varrho-k}=\sum_{n=0}^{\infty}(\varrho+k)_{n} \frac{t^{n}}{n !}(|t|<1)
$$

and interpreting in term of $(2.1)$ as a function of the form $\Phi_{v, \varrho+n, \varsigma ; \zeta}\left(\frac{z}{1-t}, \sigma, a ; p\right)$, which completes the proof of Theorem (4.2).

Theorem 4.3. The following generating function for (2.1) holds true:

$$
\begin{gathered}
\sum_{n=0}^{\infty} \frac{(\sigma)_{n}}{n !} \Phi_{v, \varrho, \varsigma ; \zeta}(z, \sigma+n, a ; p) t^{n}=\Phi_{v, \varrho, \varsigma ; \zeta}(z, \sigma, a-t ; p) \\
(p, v \geq 0, \varrho, \varsigma, \zeta \in \mathbb{C} \text { and }|t|<a ; \sigma \neq 1) .
\end{gathered}
$$

Proof. Applying (2.1) to the r.h.s of (4.11), we have

$$
\begin{aligned}
\Phi_{v, \varrho, \varsigma ; \zeta}(z, \sigma, a-t ; p) & =\sum_{k=0}^{\infty}(\varrho)_{k} \frac{B_{v}(\varsigma+k, \zeta-\varsigma ; p)}{B(\varsigma, \zeta-\varsigma)} \frac{z^{k}}{k !(k+a-t)^{\sigma}} \\
= & \sum_{k=0}^{\infty}(\varrho)_{k} \frac{B_{v}(\varsigma+k, \zeta-\varsigma ; p)}{B(\varsigma, \zeta-\varsigma)} \frac{z^{k}}{k !(k+a)^{\sigma}}\left(1-\frac{t}{k+a}\right)^{-\sigma} .
\end{aligned}
$$

Using the following binomial expansion

$$
(1-t)^{-\varrho-k}=\sum_{n=0}^{\infty}(\varrho+k)_{n} \frac{t^{n}}{n !}(|t|<1)
$$

in (4.12), we have

$$
\begin{aligned}
\Phi_{v, \varrho, \varsigma ; \zeta}(z, \sigma, a-t ; p) & =\sum_{k=0}^{\infty}(\varrho)_{k} \frac{B_{v}(\varsigma+k, \zeta-\varsigma ; p)}{B(\varsigma, \zeta-\varsigma)} \frac{z^{k}}{k !(k+a)^{\sigma}}\left\{\sum_{n=0}^{\infty} \frac{(\sigma)_{n}}{n !} \frac{t^{n}}{(k+\sigma)^{n}}\right. \\
= & \sum_{n=0}^{\infty} \frac{(\sigma)_{n}}{n !}\left\{\sum_{k=0}^{\infty}(\varrho)_{k} \frac{B_{v}(\varsigma+k, \zeta-\varsigma ; p)}{B(\varsigma, \zeta-\varsigma)} \frac{z^{k}}{k !(k+a)^{\sigma+n}}\right\} t^{n}
\end{aligned}
$$

By making the use of (2.1), we get the required result.

\section{Concluding Remark}

In this paper, we established an extension of extended Hurwitz-Lerch Zeta function and its various properties. If we consider $v=0$, then the results established here will reduce to the results studied by Parmar et al. [21]. Silmilarly, if we consider $v=0$ and then set $p=0$, we then obtained the results of generalized Hurwitz-Lerch Zeta function defined by Garg et al. [12]. 
GAUHAR RAHMAN ${ }^{1}$, KOTTAKKARAN SOOPPY NISAR ${ }^{2, \star}$ AND SHAHID MUBEEN $^{3}$

\section{REFERENCES}

[1] M. Arshad, S. Mubeen, K. S. Nisar, G. Rahman, Extended Wright-Bessel function and its properties, Commun. Korean Math. Soc. 33 (2018), No. 1, 143-155.

[2] M. Arshad, J. Choi S. Mubeen, K. S. Nisar, G. Rahman, A new extension of the Mittag-Leffler function, Commun. Korean Math. Soc. 2018, (Accepted).

[3] M. A. Chaudhry, S. M. Zubair, On a Class of Incomplete Gamma Functions with Applications, Chapman and Hall, (CRC Press Company), Boca Raton, London, New York and Washington, D. C., 2001.

[4] M. A. Chaudhry, A. Qadir, M. Rafique, S. M. Zubair,Extension of Eulers beta function, J. Comput. Appl. Math. 78 (1997) 1932.

[5] M. A. Chaudhry, A. Qadir, H. M. Srivastava and R. B. Paris, Extended Hypergeometric and Confluent Hypergeometric functions, Appl. Math. Comput., 159 (2004) 589-602

[6] J. Choi, A. K. Rathie, R. K. Parmar, Extension of extended beta, hypergeometric and confluent hypergeometric functions, Honam Mathematical J. 36 (2014), No. 2, pp. 357-385.

[7] J. Choi, D. S. Jang, and H. M. Srivastava, A generalization of the Hurwitz-Lerch Zeta function, Integral Transforms Spec. Funct., 19, (2008), 65-79.

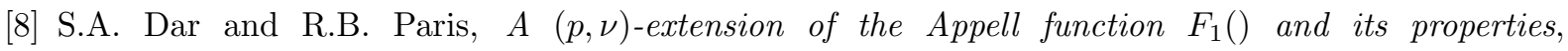
arXiv:1711.07780v1 [math.CA].

[9] S.A. Dar and R.B. Paris, $A(p, \nu)$-extension of Srivastavas triple hypergeometric function $H_{B}$ and its properties, arXiv:1711.07809v1 [math.CA].

[10] A. Erdélyi,W. Magnus, F. Oberhettinger, and F.G. Tricomi, Higher Transcendental Functions, Vol.1, McGraw-Hill, NewYork, Toronto, London, 1953.

[11] A. Erdélyi,W. Magnus, F. Oberhettinger, and F.G. Tricomi, Higher Transcendental Functions, Vol.1, McGraw-Hill, NewYork, Toronto, London, 1955.

[12] M. Garg, K. Jain, and S. L. Kalla, A further study of general Hurwitz-Lerch zeta function, Algebras Groups Geom., 25, (2008), 311-319.

[13] S. P. Goyal and R. K. Laddha, On the generalized Zeta function and the gener- alized Lambert function, Ganita Sandesh, 11, (1997), 99-108.

[14] G. Rahman, K. S. Nisar, S. Mubeen, New generalization of extended beta and hypergeometric functions, Preprints 2018, 2018020036 (doi: 10.20944/preprints201802.0036.v1).

[15] G. Rahman, K. S. Nisar, J. Choi, An extension of the Whittaker function, arXiv:1801.08032 [math.CA].

[16] G. Rahman, K. S. Nisar, M. Arshad, A new extension of Hurwitz-Lerch Zeta function, arXiv:1802.07823 [math.CA].

[17] S. Mubeen, G. Rahman, K. S. Nisar, J. Choi. M. Arshad, An extended beta function and its properties, Far East Journal of Mathematical Sciences, 102(2017), 1545-1557.

[18] S. D. Lin and H. M. Srivastava, Some families of the Hurwitz-Lerch Zeta functions and associated fractional derivative and other integral representations, Applied Mathematics and Computation, 154, (2004), 725-733

[19] E. Özergin, M. A. Özarslan, Abdullah Altn, Extension of gamma, beta and hypergeometric functions, J. Comput. Appl. Math., 235 (2011), 4601-4610.

[20] M. A. Özarslan, B. Ylmaz, The extended Mittag-Leffler function and its properties, Journal of Inequalities and Applications, 2014:85, (2014).

[21] R. K. Parmar, On a certain extension of Hurwitz-Lerch Zeta function, Analele Universităii de Vest, Timioara. Seria matematica-informatică, LII, 2(2014), 157-170.

[22] R. K. Parmar, A new generalization of gamma, beta, hypergeometric and confluent hypergeometric functions, LE MATEMATICHE Vol. LXVIII (2013) Fasc. II, pp. 3352 
A $(P, V)$-EXTENSION OF HURWITZ-LERCH ZETA FUNCTION AND ITS PROPERTIES

[23] R. K. Parmar, P. Chopra, R. B. Paris, On an Extension of Extended Beta and Hypergeometric Functions, J. Classical Anal. 11 (2017) 91106. [arXiv:1502.06200

[24] H. M. Srivastava and J. Choi, Series Associated with the Zeta and Related Functions, Kluwer, Acedemic Publishers, Dordrecht, Boston and London, 2001.

[25] H. M. Srivastava and J. Choi, Zeta and q-Zeta Functions and Associated Series and Integrals, Elsevier Science, Publishers, Amsterdam, London and New York, 2012.

[26] M. Shadab, S. Jabee and J. Choi, An extension of beta function and its application, Far East Journal of Mathematical Sciences, 103(2018), No. 1, pp 235-251. 